\title{
Geringe sexuelle Transmission von HCV
}

\section{Bei monogam lebenden Paaren, von denen einer eine chronische HCV- Infektion hat, besteht nur ein extrem geringes Risiko für eine sexuelle Übertragung der Infektion auf den Partner.}

- 500 anti-HCV-positive, HIV-negative Indexpersonen und ihre heterosexuell orientierten Langzeitpartner wurden bezüglich ihrer sexuellen Praktiken und virologischen Parameter befragt und untersucht. Bei konkordant infizierten Paaren mit demselben Genotyp erfolgte eine phylogenetische Analyse von Virusisolaten.

Im Median teilten die Partner eine gemeinsame sexuelle Aktivität über 15 Jahre hinweg mit einem sehr weiten Bereich zwischen 2 und 52 Jahren. Die mediane Anzahl von Sexualkontakten pro Monat betrug im ersten Jahr der Beziehung etwa zwölf und nahm für alle Arten der sexuellen Aktivität im Lauf der Jahre ab. Riskante Situationen wie Vaginalverkehr während der Menstruation oder Analverkehr wurden von 65\% bzw. $30 \%$ der Paare berichtet. Knapp 30\% verwendeten initial Kondome, wobei der Kondomgebrauch ebenfalls im Lauf der Jahre abnahm.

Die Gesamt-HCV-Prävalenz betrug $4 \%(n=20)$. Neun Paare hatten einen Konkordanz von Genotyp bzw. Serotyp. Die Virusisolate bei drei Paaren $(0,6 \%)$ waren nahezu identisch, was für eine Virustransmission innerhalb des Paares spricht. Auf der Basis von 8377 Personenjahren des Follow-up errechnet sich

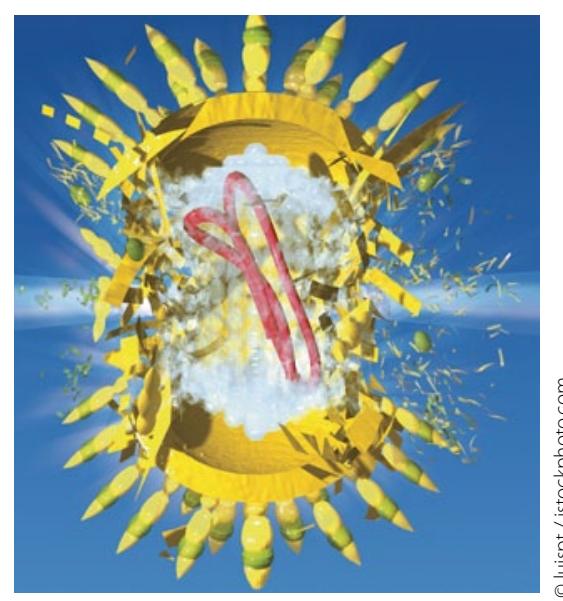

die maximale Inzidenzrate einer HCVTransmission durch sexuelle Aktivität mit $0,07 \%$ pro Jahr oder anders ausgedrückt, einer Übertragung pro 190000 Sexualkontakten.

\section{- N. A. Terrault et al.}

(Korres.: norah.terrault@ucsf.edu): Sexual transmission of hepatitis $C$ virus among monogamous heterosexual couples: The HCV partner study. Hepatology 2013;57: 881-889

\section{Kommentar}

Die Sorge, sich beim HCV-positivem Partner zu infizieren, kann dem HCV-negativen Partner angesichts dieser Zahlen genommen werden. Ähnlich günstige Ergebnisse zeigten auch Studien aus Italien und aus Deutschland in einer Gruppe von etwa 5000 mit antiD-Immunglobulin infizierten Frauen in der DDR. Dabei soll nicht verschwiegen werden, dass sich bei Schleimhautverletzungen im Einzelfall das Risiko doch erheblich erhöhen kann und nicht abzuschätzen ist.

H. S. FÜESSL =
HCV: Eine Übertragung bei 190000 Sexualkontakten.

\section{Wie gut helfen Z-Substanzen bei Insomnie?}

\begin{abstract}
Sind die Nicht-Benzodiazepine Eszopiclon, Zaleplon und Zolpidem (Z-Substanzen) wirksamer als Placebo, wenn bei einer Metaanalyse auch Daten aus Studien hinzugezogen werden, die nicht publiziert wurden?
\end{abstract}

- Ziel der Metaanalyse war es, die Wirksamkeit der Z-Substanzen Eszopiclon, Zaleplon und Zolpidem im Vergleich zu Placebo zu untersuchen und hierbei die Auswirkungen von Publikationsfehlern zu minimieren. Die Metaanalyse schloss 13 klinische Studien mit insgesamt 4378 Teilnehmern ein, die in doppelblindem placebokontrolliertem Design oben genannte Z-Substanzen bei primärer Insomnie untersucht hatten. Die Behandlungsdauer in den einge- schlossenen Untersuchungen war mindestens 14 und höchstens 180 Tage.

Für die primären Endpunkte der Schlaflatenzen zeigte die Analyse der gewichteten standardisierten mittleren Differenz sowohl für Placebo als auch die Z-Substanzen signifikante verkürzende Effekte. Bei einem Vergleich zwischen Placebo und den Z-Substanzen ergaben sich ebenfalls signifikante, aber kleine bis mittlere Unterschiede in der polysomnografisch ermittelten und subjektiven Schlaflatenz zwischen Verum und Placebo.

\section{- T. B. Huedo-Medina et al.}

Effectiveness of non-benzodiazepine hypnotics in treatment of adult insomnia: meta-analysis of datasubmitted to the Food and Drug Administration. BMJ 2012;345: e8343.

\section{Kommentar}

Die Verschreibung von Benzodiazepinen zur pharmakologischen Behandlung von Insomnien hat zwar abgenommen, der Gebrauch von Hypnotika insgesamt ist aber durch die Markteinführung der Z-Substanzen unverändert hoch geblieben. Dies mag auch darin begründet sein, dass die Z-Substanzen in der Wahrnehmung von Hausärzten sicherer und effektiver als Benzodiazepine sind, wofür es keine überzeugende Evidenz gibt. Vor diesem Hintergrund ist diese Metaanalyse von großem Interesse, spricht sie doch dafür, dass ein nicht unerheblicher Teil der Wirkung der Z-Substanzen durch einen Placeboeffekt vermittelt wird.

P. C. BAIER = 